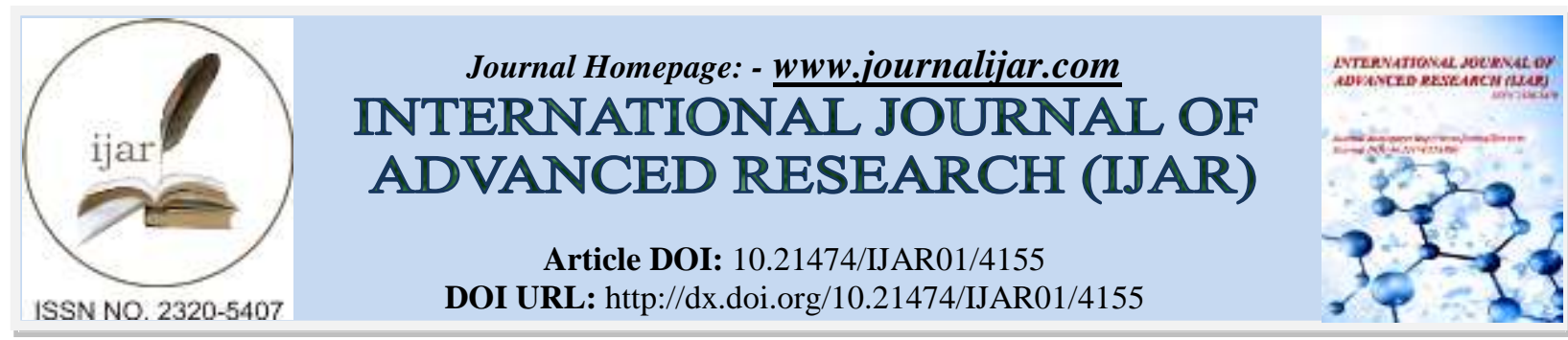

RESEARCH ARTICLE

\title{
MAGNETIC RESONANCE SPECTROSCOPY AND THE METABOLIC FINGERPRINT OF MALIGNANT MAXILLOFACIAL TUMORS. A DIAGNOSTIC ACCURACY STUDY.
}

\section{Salma Belal Eiid ${ }^{1}$, Mohamed Khalifa Zayet ${ }^{2}$ and Mushira Mohamed Dahaba ${ }^{3}$.}

1. Assistant lecturer of Oral and Maxillofacial Radiology, Faculty of Oral and Dental Medicine, Cairo University; Cairo, Egypt.

2. Ass. Professor of Oral and Maxillofacial Radiology, Faculty of Oral and Dental Medicine, Cairo University; Cairo, Egypt.

3. Professor of Oral and Maxillofacial Radiology, Faculty of Oral and Dental Medicine, Cairo University; Cairo, Egypt.

\section{Manuscript Info}

[.........................

Manuscript History

Received: 06 March 2017

Final Accepted: 12 April 2017

Published: May 2017

Key words:-

Magnetic resonance spectroscopy -

Choline - Lactate - Diagnostic accuracy

\section{Abstract}

Objective: The purpose of this study was to define the malignant metabolic profile of maxillofacial lesions using Magnetic resonance spectroscopy.

Subjects and Methods: Twenty-one patients with maxillofacial lesions were enrolled in this study through consecutive sampling.Single-voxel MRS was performed using a spin echo (point resolved spectroscopy) sequence. Qualitative and semi-quantitative analyses were performed to assess the choline and lactate peaks in benign and malignant lesions.

Results: Statistical analysis showed that there was a significant difference between the mean of both metabolites in benign and malignant entities. The diagnostic accuracy of lactate was higher than that of choline. Threshold values of $>0.125$ and 0.336 for lactate and choline were extracted in a trial to differentiate between both entities.

Conclusion: Magnetic resonance spectroscopy is a state-of-art in the diagnostic arena and manifested itself as a key player in the differentiation process of maxillofacial tumors.

Copy Right, IJAR, 2017,. All rights reserved.

\section{Introduction:-}

Up to date the histopathological diagnosis is the gold standard in the diagnostic process of benign and malignant maxillofacial lesions. However, this is not always an easy task and sometimes this process becomes very daunting. Biopsy can be an invasive procedure and may cause damage of nearby vital structures. Occasionally biopsy may become fatal in vascular lesions and the worst scenario is the histopathological resemblance between some malignancies with their benign counterparts (Mohajerani et al., 2009).

Moreover, clinical and radiographic finding can be a well-established guideline to differentiate between benign and malignant entities. Nevertheless, some malignant tumors can mimic benign tumors clinically and radiographically and vise versa (Kalele et al., 2016).Regarding the multifaceted nature of magnetic resonance imaging (MRI), there is still no consensus that conventional MRI can precisely differentiate benign from malignant lesions (Rimondi et al., 2016).Therefore, there is still a significant gap in the diagnostic field and imaging modalities should be modernized 
and upgraded to meet this need. This is done through integrating the functional or biological techniques such as magnetic resonance spectroscopy (MRS)(Kauppinen and Peet, 2011).

MRS is one of the viable methods that can non-invasively examine different metabolites present in different diseases. Cancer is known for its distinctiveness in producing specific metabolites because of the rapid turn over of cells and the presence of hypoxia with concurrent necrosis formation. These changes can be probed using the power of a metabolic imaging technique as MRS (García-Figueiras et al., 2016). Therefore, this study was conducted to assess the capability of MRS in adequately defining the metabolic profile of malignant tumors.

\section{Materials and Methods:-}

According to the Code of ethics, this diagnostic accuracy study was undertaken after the approval of the Research Ethics Committee.

\section{Patient Population:-}

Twenty-one patients out of twenty-three with lesions in the craniofacial area were enrolled in this study through consecutive sampling (one was excluded because of incomplete histopathological confirmation and the other showed high artifact levels with degraded image quality in MRI scan). The patients were collected from the Oral and Maxillofacial Radiology outpatient clinic, Cairo University or from Kasr El-Aini Hospital. The population comprised of nine males and twelve females with twenty-two lesions between the age range of 7 to 76 and mean age of 33.5 years.

A predetermined list of inclusion and exclusion criteria was planned, where all maxillofacial lesions with radiolucent or mixed appearance in radiographic examination of size greater than $1 \mathrm{~cm}^{3}$ were included in this study. However, exclusion of candidates was based on the presence of contraindication to MRI as those with pacemakers, pregnant females or if artifacts degraded the quality of the images, the data were excluded from further analysis.

\section{MRI Data Acquisition:-}

MRI examination was performed on a 1.5T superconducting MR system (Gyroscan, Intera, Philips - Netherlands) at Kasr El-Aini Hospital, Cairo University. A neurovascular coil was adequately adjusted for each patient to cover the head and neck area.

\section{Magnetic Resonance Spectroscopy (MRS):-}

Single-voxel MRS was performed using a spin echo (point resolved spectroscopy - PRESS) sequence with water suppression using Neurovascular coil. The sequence parameters utilized were $2000 \mathrm{~ms}$ TR, $144 \mathrm{~ms}$ TE, and NEX 128. The voxel was placed on the tumor based on axial, coronal, and sagittal images (T2 weighted images). Voxel size drawn was not smaller than $1 \mathrm{~cm} \times 1 \mathrm{~cm} \times 1 \mathrm{~cm}$ in all the cases, to increase the coverage of the lesion as large as possible without inclusion of any heterogeneous tissues such as calcifications, bony septa, or fatty tissues (Fig 1).

\section{Data Post-Processing:-}

The assessment of data was done blindly without knowing the results of histopathology. Data was analyzed qualitatively and quantitatively:

\section{Qualitative analysis:-}

Presence or absence of metabolites peaks (Choline "Cho" and Lactate "Lac") was checked.

\section{Semi-Quantitative analysis:-}

The value of metabolite peak height was extracted using the standard software provided by the manufacturer (Fig 1).

\section{Histopathological findings:-}

Incisional or excisional biopsies were taken for the lesions directly after scanning. Then, a pathologist interpreted the lesions without knowing the results of MR imaging techniques.

\section{Statistical analysis:-}

Statistical analysis was performed with SPSS 20.0 (Statistical Package for Scientific Studies) for windows (SPSS, Inc., an IBM Company USA). The mean and standard deviations of benign and malignant entities were calculated for Cho and Lac peaks. The receiver operating characteristic (ROC) curve was plotted with the $95 \%$ confidence 
intervals to evaluate the diagnostic capability of both parameters by calculating Areas under the ROC curves (AUC). Moreover, best cut-off values were determined using the Youden Index J and displayed using scatter dot plots with their corresponding sensitivity and specificity. Lastly, Likelihood ratios were calculated from the sensitivity and specificity of each parameter.

\section{Results:-}

In this current study, the population was categorized according to the histopathological nature into benign and malignant processes. Lesions in both categories were listed in Table 1.

\section{Descriptive findings:-}

All lesions in this study revealed a choline peak. Regarding lactate, only four benign lesions showed a lactate peak, and all malignancies except one showed a lactate peak. It is clear from the data in Table 2that values in malignant sample have a significant difference ( $\mathrm{p}<0.01$ in all cases) when compared to benign group using unpaired t-test. Lac values were much smaller in benign $(0.062 \pm 0.095)$ than in malignant ones $(0.672 \pm 0.543)$. A similar trend appeared in the Cho data, where benign showed a mean of $0.229 \pm 0.078$ and malignancy of $0.515 \pm 0.376$.

\section{Diagnostic Accuracy (The ROC curve):- ROC Curves and AUCs analyses:-}

The ROC curves for both metabolites were plotted with the 95\% confidence intervals 'CI' (dotted lines) (Fig 2 and 3). The higher the AUC, the better is the diagnostic test. The data in Table 3 shows that Lac yielded higher AUC $(0.908 \pm 0.0701, \mathrm{CI}=0.707$ to $0.989, \mathrm{p}<0.0001)$, than $\mathrm{Cho}(0.746 \pm 0.133, \mathrm{CI}=0.517$ to $0.905, \mathrm{p}=0.0649)$.

\section{Cut-off values and the calculation of Likelihood ratios:-}

The cut-off values were calculated according to the Youden Index $\mathbf{J}$ method, summarized in Table 4 with their corresponding sensitivity and specificity. Likelihood ratio calculation is a paramount statistical measure in diagnostic accuracy studies. Likelihood ratios (Table 4) measure the likelihood of the disease and are a combination of positive and negative LRs (LR+ and LR-).

LR+ = Sensitivity / $1-$ Specificity

LR- = 1 - Sensitivity / Specificity

The positive LR+ indicates better diagnostic outcomes if the value is farther from 1. On the other hand, the smaller the -LR is the more is the suggestion of the absence of the disease.

\section{Discussion:-}

MRS can be integrated to the conventional MRI scan to non-invasively unveil certain biological aspects of tumors through probing different metabolites within tissues. Therefore, it can give an indication of the behavior of the underlying disease (Arda and Aydın, 2016).

It is rare in literature to apply MRS to maxillofacial and jaw lesions due to the challenges in obtaining adequate quality of MR spectra because of small irregular sizes of lesions and the low SNR of the images. Nevertheless, all spectra were of good quality conforming with what Abdel Razek and Poptani 2013 and Varoquaux et al 2015 had presumed that failure of the spectroscopy examination in general is detected when a spectrum containing only noise without any identifiable metabolite peaks and spectra are classified of high quality when the separation of resonance of important metabolites in a tumor is an easy task.

Small metabolic peaks unfortunately can be totally obscured by misplacement of voxel (Deshmukh et al., 2014). To exclude any inconsistencies in the present study, T2 sagittal, coronal and axial WIs were used as a guide for voxel placement according to what $\mathrm{Zi}$-hua et al 2009 and Chawla et al 2009have suggested. PRESS was used according to multiple recommendations from different authors as Yu et al 2007, Subhawong et al 2012, Abdel Razek \& Poptani 2013 and Deshmukh et al 2014.

Choline and lactate were assessed, since they are the most sought metabolites in tumor assessment as declared by Abdel Razek and Poptani 2013 and García-Figueiras et al 2016.Although, lactate is an important contributor in the spectral analysis of a tumor, however, most of the studies in literature focused on the sole presence of the choline whether quantitative or qualitative. This problem was relatively solved in this study and lactate amount was successfully measured. 
It is known that the observed metabolite peaks change depending on the length of TE. An intermediate TE of 130$150 \mathrm{~ms}$ is always needed to observe the metabolite choline. Moreover, long or intermediate TE should be considered for lactate evaluation (Yu et al., 2007, Deshmukh et al., 2014, and García-Figueiras et al., 2016).Maheshwari et al in 2000stated that it is not necessary to perform protocols using both intermediate and long TEs in a clinical setting. Therefore, only one TE of $144 \mathrm{~ms}$ was optimized in this study in hand.

In our study, according to the qualitative assessment of choline peaks, all benign and malignant tumors showed a choline signal. Although most of peaks of benign lesions were smaller than that of malignant, however, some just overlapped. This coincided with a study performed on salivary gland tumors by King et al 2005, where Cho was present in all benign and malignant tumors. The mean of the height of Cho of benign vs. malignant tumors was 0.229 and 0.515 respectively with a cutoff value of 0.336 , where lesions with higher Cho should be considered malignant.

Regarding the qualitative assessment of lactate peaks all malignant tumors showed lactate signal except one (90\%), which was nasopharyngeal carcinoma. On the other hand, only 4 out of $12(33.3 \%)$ benign tumors showed a lactate peak. Infection showing lactate peak can be interpreted as an expected phenomenon because of necrotic tissue present. However, the other 3 benign tumors (dentigerous - ameloblastoma - brown tumor of HPT) presenting lactate in their metabolic profile is rather very intriguing and further investigations are needed to assess the presence of lactate in benign tumors. Semi-quantitative assessment of Lactate showed a statistically significant difference between the mean of benign (0.06) and malignant (0.67) tumors.

ROC analysis showed that lactate had higher the AUC than choline, i.e., higher diagnostic accuracy and Youden Index $\mathrm{J}$ proposed a cut-off value of $>0.125$. Although, choline didn't show statistically diagnostic significance, nevertheless, individual statistical measures as sensitivity, specificity and likelihood ratios (LR) should be interpreted as well.

Choline had larger LR+ $(\infty)$, i.e., the highest in ruling in malignancy, meaning that any lesion more than 0.336 (calculated cut-off value) is supposed to be a malignancy. No benign tumors were above that value in this study (no false positive $-100 \%$ specificity). However, because of the selected threshold value and the major discrepancies of choline in benign and malignant tumors, it had high LR - of 0.5 with high false negative rate, that's why they represented $70 \%$ sensitivity.

Lactate showed lower LR+ of 5.4 with lower capability in ruling in malignancy with 2 false positive and specificity of $83.3 \%$ at $>0.125$ threshold value. Nevertheless, lactate had rather stronger capability in suggesting the absence of the disease or disease exclusion because of lower LR- of 0.12 with only one false negative and $90 \%$ sensitivity. Therefore, Laccan be used as a diagnostic parameter in ruling in malignancies without jeopardizing much the chance of missing some.

Subhawong et al in 2012 performed a pooled analysis of MRS studies of musculoskeletal lesions and proved a strong association between the presence of a choline peak and malignancy with a sensitivity of $88 \%$ and a specificity of $68 \%$. Nevertheless, some benign lesions seemed to show choline in their metabolic profile. Likewise, Zi-hua et al in 2009 revealed that hypercellular benign tumors of rapid and aggressive behavior can show a choline peak. Similary, Yu et al 2007justified that an increased Cho signal in some benign tumors might be due to hyper cellularity, and added that hyper vascularity can have the same effect. This declaration rationalizes why both cases of angiofibromas in our study, although fluid-filled but showed Cho signals at the top of the benign range.

The highest choline peaks in this sample were 1.16 and 1.10 related to nasopharyngeal carcinoma and neuroblastoma. These values are very high when compared to the whole sample, which highlighted the notion proposed by Agarwal and coworkers in 2014 which, suggested that the degree of choline rise was correlated to the histologic aggressiveness of the tumor (highly cellular and less differentiated tumors).

Although, the above notions seem flawless and how malignant behavior can be easily manifested in MRS. Unfortunately, some malignancies just mimicked benign regarding the low value they acquired. Lowest choline values in malignancies were central SCC, tongue SCC, and malignant lymphoma in the maxilla with values 0.025 , 0.196 , and 0.150 respectively. These three benign mimickers were below the cutoff value suggested. 
False negative results has been highly encountered in several previous MRS studies. Wang et al 2004, Zi-hua et al 2009, and Agarwal et al 2014 clarified that improper voxel placement within a bony or calcified areas of the tumors affected their results. Extensive care was taken during voxel placement in this study. However, this was not quite easy in this sample, where some of the maxillofacial tumors were relatively small with inevitable inclusion of calcifications or septa that degraded Cho calculation.

Other than voxel placement, false negative results or benign mimickers might increase due to the inclusion of necrosis with subsequent diagnostic challenges encountered. This was suggested by Jansen et al in 2012 , where areas of dense cell proliferation are associated with high choline concentrations, due to a high membrane turnover, nevertheless, highly necrotic regions can give very low choline peaks. Therefore, there must be other variables such as hypoxia to rely on during MRS assessment rather than cell proliferation(Hafez et al., 2016).Therefore, in this study in hand, lactate was also assessed, since, it is capable of measuring the degree of hypoxia, which is related to the degree of tumor differentiation and the amount of necrosis present (Abdel Razek and Poptani 2013 and Hafez et al., 2016).

The level of tumor lactate has been shown to correlate negatively with tumor oxygenation and, additionally, with patient outcome (Agarwal.et al 2014). This was somehow assessed herein, histopathological reports of cases with very high lactate peaks, as chondrosarcoma and osteosarcoma were grade III tumors.

Therefore, the full MRS profile of the malignancy fingerprint can be decoded as high choline and lactate peaks, whereas benign lesions fingerprint is reversed with low choline and no lactate peaks. According to this study conditions and limitations, one of the major shortcomings of this study is the small sample size along with the diverse types of tumors that tend to affect the head and neck area. This heterogeneity in the small sample may negatively have influenced the performance of the technique.

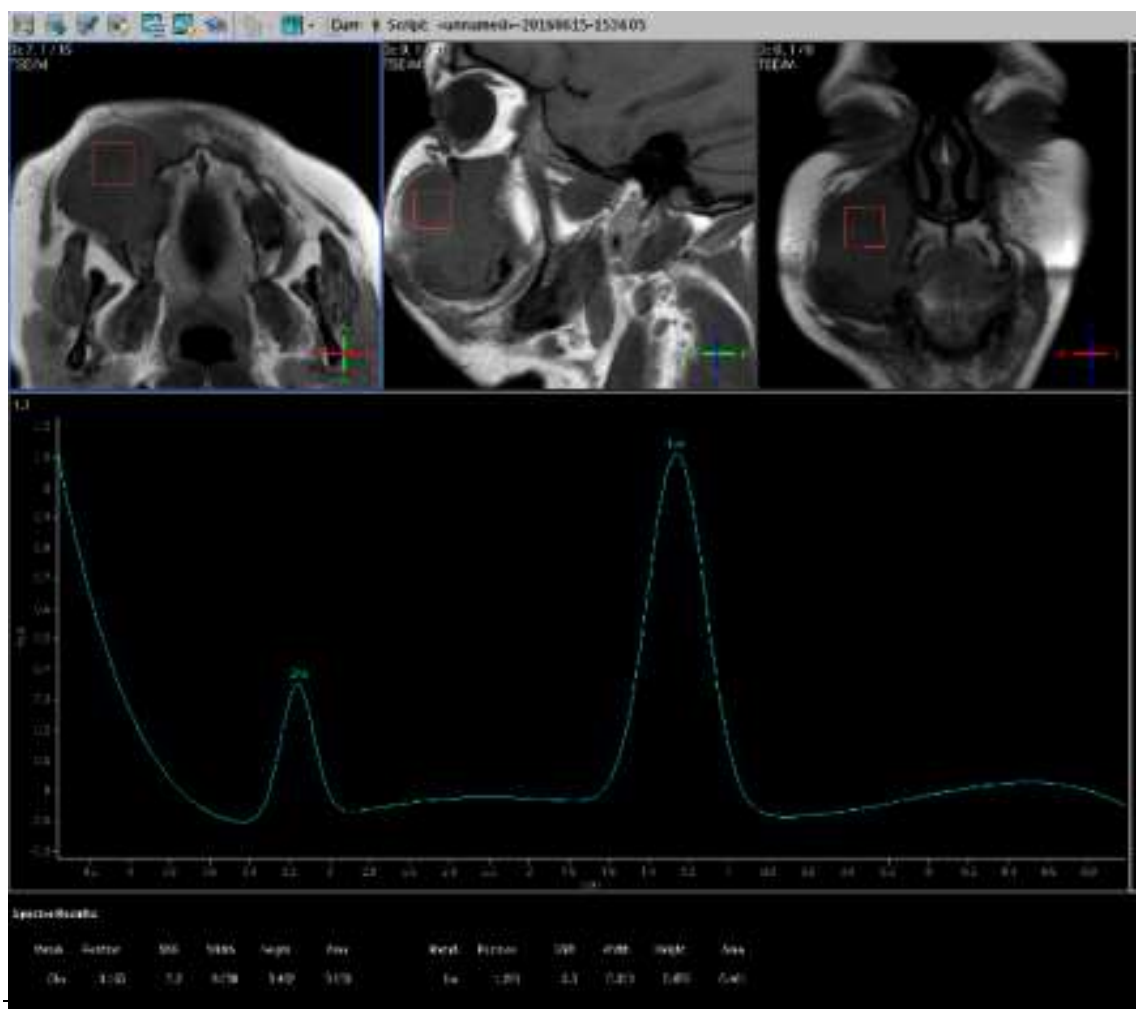

Figure 1:- A 29 years old female patient with an ameloblastic fibrosarcoma in the maxilla. The voxel was adequately drawn using the three planes without inclusion of any heterogeneous tissues (above). MR spectrum displayed the presence of both peaks with the automatic calculation of their height values below (below). 


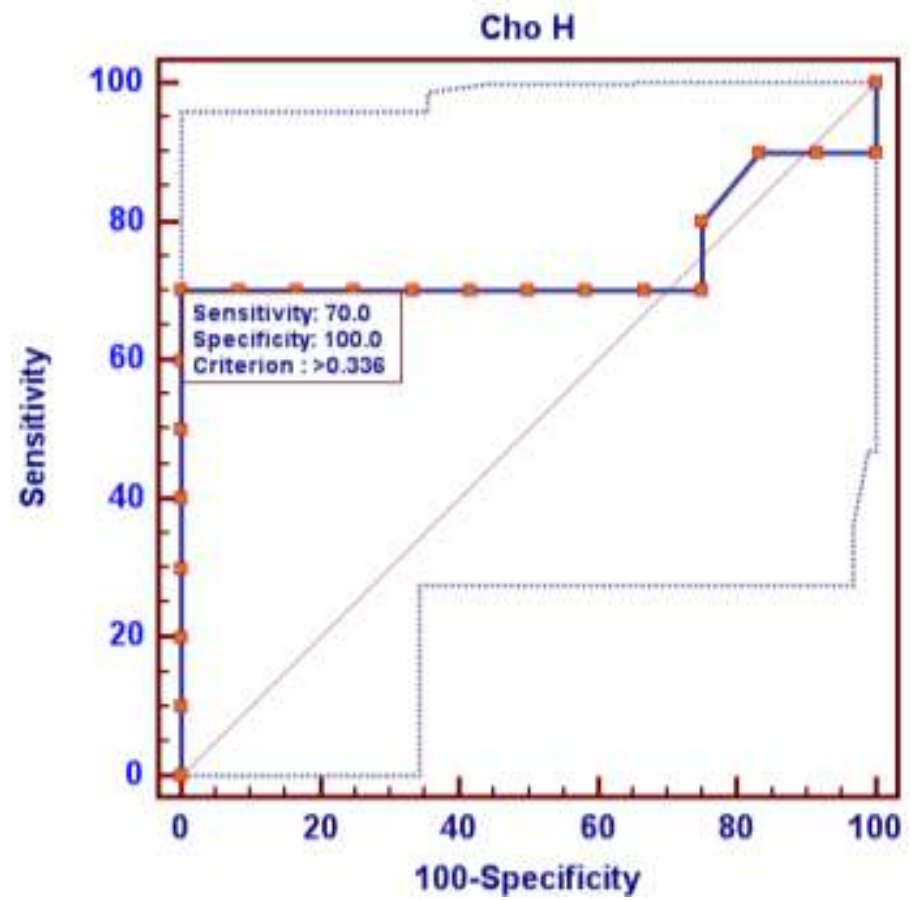

Figure 2:- ROC curve for Choline metabolite

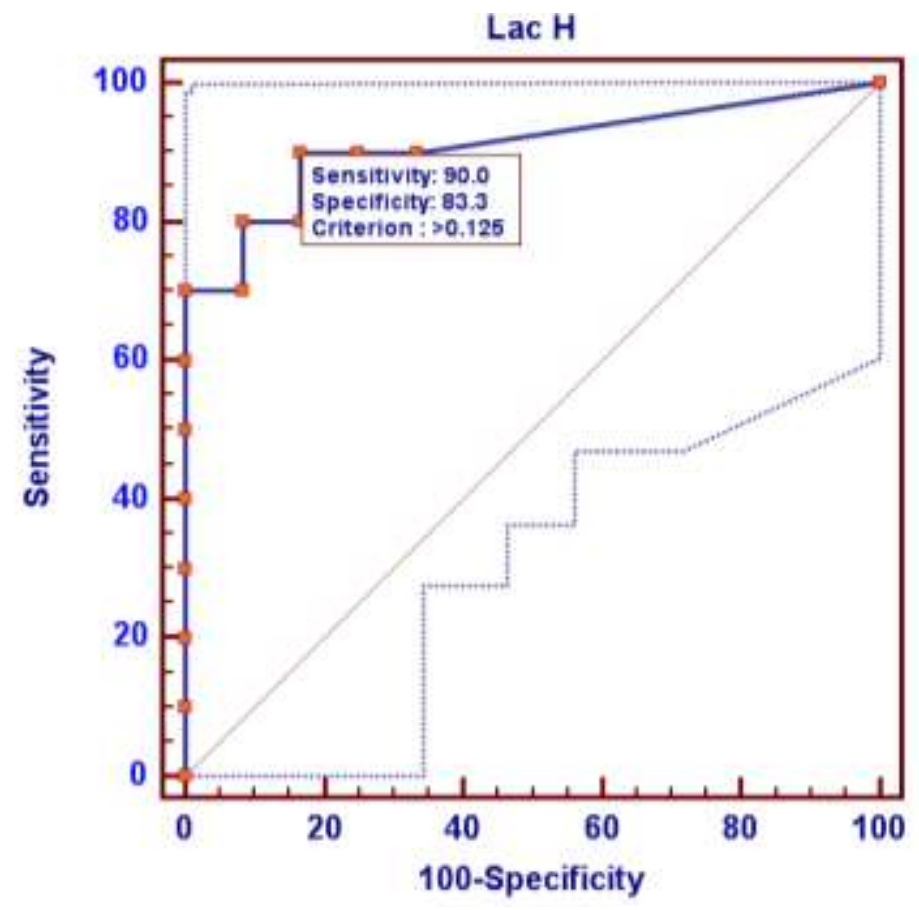

Figure 3:- ROC curve for Lactate metabolite 
Table 1:- Histopathological entities in both categories:

\begin{tabular}{|c|c|c|c|c|}
\hline Lesion type & Pathology & No. & \multicolumn{2}{|l|}{ Location } \\
\hline Malignancy (10) & $\begin{array}{l}\text { Malignant Lymphoma } \\
\text { Carcinoma } \\
\text { Osteosarcoma } \\
\text { Metastasis from breast } \\
\text { Neuroblastoma } \\
\text { Chondrosarcoma } \\
\text { AmeloblasticFibrosarcoma }\end{array}$ & $\begin{array}{l}1 \\
3 \\
2 \\
1 \\
1 \\
1 \\
1\end{array}$ & \multicolumn{2}{|c|}{$\begin{array}{l}\text { Maxilla } \\
\text { Pharynx-Tongu-Mandible } \\
\text { Maxilla } \\
\text { Mandible } \\
\text { Sinonasal area } \\
\text { Mandible } \\
\text { Maxilla }\end{array}$} \\
\hline Benign (12) & \multirow{3}{*}{$\begin{array}{l}\text { Infection } \\
\text { Ameloblastoma } \\
\text { KCOT } \\
\text { Juvenile Aggressive } \\
\text { Fibromatosis } \\
\text { Angiofibroma } \\
\text { Odontogenic Myxoma } \\
\text { Đentigerous Cyst. } \\
\text { Brown tumor of HipT }\end{array}$} & 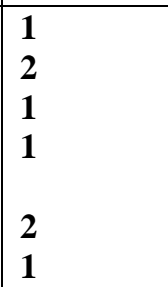 & \multirow{3}{*}{\multicolumn{2}{|c|}{$\begin{array}{l}\text { Maxilla } \\
\text { Mandible } \\
\text { Mandible } \\
\text { Medial Pterygoid muscle } \\
\text { encroaching the Ramus } \\
\text { Naso-ethmoidal area } \\
\text { Maxilla } \\
\text { Maxilla } \\
\text { ChO } \\
\text { Bandible - Ramus } \\
\text { Bentgn }\end{array}$}} \\
\hline & & 1 & & \\
\hline & & Malignant & & \\
\hline Number of values & Arreriovenous matrom & $1 \rho$ & \multirow{2}{*}{$\begin{array}{l}\text { Mandible } \\
0.2288\end{array}$} & 10 \\
\hline Mean & 0.06208 & 0.6718 & & 0.515 \\
\hline Std. Deviation & 0.09476 & 0.5428 & 0.07845 & 0.3759 \\
\hline Unpaired t-test & \multicolumn{2}{|l|}{ **** } & \multicolumn{2}{|l|}{$* *$} \\
\hline $\mathrm{p}$ value & \multicolumn{2}{|l|}{0.0008} & \multicolumn{2}{|l|}{ 0.0089 } \\
\hline
\end{tabular}

KCOT: Keratocystic odontogenic tumor, HPT: Hyperparathyroidism

Table 2:- Descriptive statistics of data classified according to malignancy

Table 3: Comparison of ROC curves' AUC:

\begin{tabular}{|l|l|l|l|l|}
\hline & AUC & SE & $95 \%$ CI & P value \\
\hline Cho & 0.746 & 0.133 & 0.517 to 0.905 & 0.0649 \\
\hline Lac & 0.908 & 0.0701 & 0.707 to 0.989 & $<0.0001$ \\
\hline
\end{tabular}

${ }^{a}$ DeLong et al., 1988; SE: Standard Error

${ }^{\mathrm{b}}$ Binomial exact

Table 4:- Cut-off values, corresponding sensitivity and specificity for each parameter and the calculated LRs:

\begin{tabular}{|l|l|l|l|l|l|l|l|l|l|}
\hline & Cut-off & Sensitivity & $\mathbf{9 5 \%}$ CI & Specificity & $\mathbf{9 5 \%}$ CI & +LR & 95\% CI & -LR & 95\% CI \\
\hline Cho & $>\mathbf{0 . 3 3 6}$ & $\mathbf{7 0 . 0 0}$ & $34.8-93.3$ & $\mathbf{1 0 0 . 0 0}$ & $73.5-100.0$ & $\infty$ & & 0.30 & $0.1-0.8$ \\
\hline Lac & $>\mathbf{0 . 1 2 5}$ & $\mathbf{9 0 . 0 0}$ & $55.5-99.7$ & $\mathbf{8 3 . 3 3}$ & $51.6-97.9$ & 5.40 & $1.5-19.5$ & 0.12 & $0.02-0.8$ \\
\hline
\end{tabular}

Cut-off values: values associated with Youden Index J, +LR: positive likelihood ratio; - LR: negative likelihood ratio

Moreover, according to the problems encountered, it is important to realize that choline might have no significant diagnostic capability because of the study limitation, where single voxel was used without considering the undeniable negative effect of tumor hypoxia and necrotic tissue formation. Therefore, it can be deduced that it is either to use multi-voxel MRS, to avoid the interpretation of one voxel with a misleading result or assess the two metabolites Cho and Lac to guide through the interpretation process.

Acknowledgment: No source of funding. 


\section{References:-}

1. Abdel Razek, A. A. K., \& Poptani, H. (2013). MR spectroscopy of head and neck cancer. Eur J Radiol, 82(6), 982-9. doi:10.1016/j.ejrad.2013.01.025

2. Agarwal, S., Kundu, Z., Kumar, S., \& Sangwan, S. (2014). Single voxel 1 H magnetic resonance spectroscopy in the diagnosis of musculoskeletal mass lesions. Clinical Cancer Investigation Journal, 4(1), 66. doi:10.4103/2278-0513.125800

3. Arda, K., \& Aydın, H. (2016). Proton Mr Spectroscopy, Fundamental Physics and Clinical Applications. Austin Oncol, 1(1), 1002.

4. Chawla, S., Kim, S., Loevner, L. A., Quon, H., Wang, S., Mutale, F., ... Delikatny, E. J. (2009). Proton and Phosphorous MR Spectroscopy in Squamous Cell Carcinomas of the Head and Neck. Acad Radiol, 16(11), 1366-1372. doi:10.1016/j.acra.2009.06.001.Proton

5. Deshmukh, S., Subhawong, T., Carrino, J., \& Fayad, L. (2014). Role of MR spectroscopy in muculoskeletal imaging. Indian J Radiol Imaging, 24(3), 210-216. doi:10.4103/0971-3026.137024

6. García-Figueiras, R., Baleato-González, S., Padhani, A. R., Oleaga, L., Vilanova, J. C., Luna, A., \& Gómez, J. C. C. (2016). Proton magnetic resonance spectroscopy in oncology: The fingerprints of cancer? Diag Interv Radiol, 22(1), 75-89. doi:10.5152/dir.2015.15009

7. Hafez, H., Elmoneim, B., Fawzy, T., \& Omar, S. (2016). The role of proton magnetic resonance spectroscopy in grading of brain gliomas. Menoufia Med J, 29(1), 136-140. doi:10.4103/1110-2098.179004

8. Jansen, J. F. ., Schöder, H., Lee, N. Y., Stambuk, H. E., Wang, Y., Fury, M. G., ... Dave, A. S.-- (2012). Tumor Metabolism and Perfusion in Head and Neck Squamous Cell Carcinoma: Pretreatment Multimodality Imaging with 1 H- Magnetic Resonance Spectroscopy, Dynamic Contrast- Enhanced MRI and 18 F-FDG PET. Int J Radiat Oncol Biol Phys, 82(1), 299-307. doi:10.1016/j.ijrobp.2010.11.022.Tumor

9. Kalele, K. P., Kulkarni, N., Patil, K. P., Nayyar, A. S., Kulkarni, M., \& Atram, P. (2016). Retrospective Analysis of Discrepancies between Clinical and Histopathological Diagnoses in Head and Neck Lesions : An Institutional Study with 10 Years Database. J. Oral Maxillofac. Pathol, 7(1), 657-664.

10. Kauppinen, R. a., \& Peet, A. C. (2011). Using magnetic resonance imaging and spectroscopy in cancer diagnostics and monitoring: preclinical and clinical approaches. Cancer Biol Ther, 12(8), 665-679. doi:10.4161/cbt.12.8.18137

11. King, A. D., Yeung, D. K. W., Ahuja, A. T., Tse, G. M. K., Yuen, H. Y., Wong, K. T., \& Hasselt, A. C. Van. (2005). Salivary Gland Tumors at in Vivo Proton MR Spectroscopy. Radiology, 237(2), 563-569.

12. Maheshwari, S. R., Mukherji, S. K., Neelon, B., Schiro, S., Fatterpekar, G. M., Stone, J. a, \& Castillo, M. (2000). The choline/creatine ratio in five benign neoplasms: comparison with squamous cell carcinoma by use of in vitro MR spectroscopy. AJNR. Am J Neuroradiol, 21(10), 1930-1935. Retrieved from http://www.ncbi.nlm.nih.gov/pubmed/11110549

13. Mohajerani, H., Mosalman, M., Mohajerani, S. A., \& Ghorbani, Z. (2009). Frequency of Giant Cell Lesions in Oral Biopsies. J Dent (Tehran), 6(4), 193-197.

14. Rimondi, E., Benassi, M. S., Bazzocchi, A., Balladelli, A., Facchini, G., Rossi, G., ... Vanel, D. (2016). Translational research in diagnosis and management of soft tissue tumours. Cancer Imaging, $16(1), 13$. doi:10.1186/s40644-016-0071-7

15. Subhawong, T. K., Wang, X., Durand, D. J., Jacobs, M. a, Carrino, J. a, Machado, A. J., \& Fayad, L. M. (2012). Proton MR spectroscopy in metabolic assessment of musculoskeletal lesions. AJR. Am J Roentgenol, 198(1), 162-72. doi:10.2214/AJR.11.6505

16. Varoquaux, A., Fur, Y. le, Imperiale, A., Reyre, A., Montava, M., Fakhry, N., ... Taïeb, D. (2015). Magnetic Resonance Spectroscopy of paragangliomas: new insights into in vivo metabolomics. Endocr Relat Cancer, 22(4), M1-M8. doi:10.1530/ERC-14-0411.Persistent

17. Wang, C., Li, C., Chien, S., Liu, G., \& Tsai, K.-B. (2004). Characterization of Bone and Soft-Tissue Tumors with in Vivo $1 \mathrm{H}$ MR Spectroscopy: Initial Results 1. Radiology, 232(2), 599-605.

18. Yu, Q., Yang, J., Wang, P., Shi, H., \& Luo, J. (2007). Preliminary assessment of benign maxillofacial and neck lesions with in vivo single-voxel $1 \mathrm{H}$ magnetic resonance spectroscopy. Oral Surg Oral Med Oral Pathol Oral Radiol Endod, 104(2), 264-270. Retrieved from http://search.bvsalud.org/portal/resource/en/mdl-17630099

19. Zi-hua, Q. I., Chuan-fu, L. I., Zhen-feng, L. I., Kai, Z., Qian, W., \& De-xin, Y. U. (2009). Preliminary study of 3T 1H MR spectroscopy in bone and soft tissue tumors. Chin Med J, 122(1), 39-43. doi:10.3760/cma.j.issn.0366-6999.2009.01.008 